Deichmüller, nachgewiesen haben, eine merkbare Eigenbewegung nicht vorhanden, und es fielen die gefundenen Grössen also allein den Vergleichsternen zu. Nimmt man nun sämmtliche $\varepsilon$ als verschwindend an, so ergiebt sich von Neuem, dass $\left[\alpha-1 / 2\left(\alpha_{1}+\alpha_{2}\right)\right]$ sich nicht von $\pi$ trennen lässt. Man hat dann wie früher zu verfahren und erhält so

$x=+0.185 ; \quad \pi=0.02 ; \quad 2 \alpha-\alpha_{1}-\alpha_{2}=+0.33$ und die unter $(B-R)_{\text {II }}$ mitgetheilte Darstellung.

Da der Beobachter selbst die zweite und letzte Beobachtung für weniger sicher erklärt, ist es völlig zweifelhaft, welcher Annahme man am meisten vertrauen soll. In jedem Falle dürfte mit unzweifelhafter Sicherheit hervorgehen, dass die vorliegenden Beobachtungen auf die Eigenbewegungen keinen sicheren Schluss erlauben, dass ferner eine verschwindende Parallaxe sich mit ihnen vollkommen verträgt und die Einwirkung der Extinction sich noch relativ sicher bestimmen lässt, aber von der Einwirkung der Parallaxe kaum getrennt werden kann. Die Zahlen bedeuten, wenn von einer Färbung des betreffenden Sterns in obigem Sinne gesprochen wird,

München, I902 Mai 8. dass der Stern $B x$ um rund $5 \circ \mu \mu$ röther ist als $A$, die Nova um rund $3 \circ \mu \mu$ weniger roth als $A$ und um $80 \mu \mu$ weniger als $B x$. Das erstere Resultat, welches etwas sicherer verbürgt zu sein scheint, stimmt mit der Bemerkung Herrn Hartwig's überein, nach welcher ihm der Stern $B x$ röthlich erschienen ist. Derselbe hätte jedenfalls von der Beobachtung ausgeschlossen werden müssen, wenn man auf den Einfluss der Dispersion keine Rücksicht nehmen wollte.

Im Uebrigen bemerke ich nochmals ausdrücklich, dass die angeführten Zahlen mehr des Beispiels wegen und nicht um sichere Resultate zu geben angefuhtt worden sind, denn zur Ableitung solcher müssten die Beobachtungen zahlreicher und iber verschiedene Stundenwinkel vertheilt sein. Man darf aber doch aussprechen, dass selbst sehr genaue Heliometerbeobachtungen, an wenig zahlreichen Abenden angestellt, unter Umständen kleine Parallaxen, also solche, die kleiner als etwa o"! I sind, abzuleiten nicht gestatten und dass die Dispersion der Luft hierbei ein wesentliches Hinderniss bildet, das nur durch die Beobachtung in sehr verschiedenen Stundenwinkeln eventuell weggeräumt werden kann.

\title{
Zusatz zu meiner Notiz: „Versuch einer Erklärung der Entstehung und der Bewegung der Nebelhülle, welche die Nova Persei umgiebt", Astr. Nachr. 3765.
}

\author{
Von $\mathcal{F}$. Wilsing:
}

In den Astr. Nachr. Nr. 3790 hat Herr Halm eine Bemerkung gemacht, welche die Beantwortung der von mir gestellten Frage betrifft, ob die Annahme einer gleichförmigen Ausdehnung der die Nova unmittelbar umgebenden gasförmigen Hülle in radialer Richtung zur Erklärung der Doppellinien im Spectrum einen wesentlichen Beitrag liefern kann. Die Richtigkeit dieser Annahme erschien mir nicht wahrscheinlich, weil sich die Strahlung der äusseren Theile der Chromosphäre, welche nach der Hypothese die hellen Linien giebt, über die dunklen Bänder legt, welche von der Absorption der in Richtung des Visionsradius vor dem Kern befindlichen Gasmassen herrührent. Herr Halm bemerkt nun sebr zutreffend, dass der nach Violett gelegene Rand der Absorptionslinien nicht durch die Emission der seitlich gelegenen Theile der Chromosphäre aufgehellt werden kann, übersieht aber dabei, dass es sich im vorliegenden Falle nur um die Erklärung der breiten diffusen Absorptionsbänder handeln kann, wie sie für das erste Stadium des Novaspectrums charakteristisch zu sein scheinen. Bei der Nova Aurigae waren die dunklen Bänder sogar merklich von gleicher Breite, wie die hellen Bänder. Die Voraussetzung für die Sichtbarkeit der auf das intensive, vom Kern herrührende continuirliche Spectrum projicirten hellen Bänder bedingt die Annahme eines beträchtlichen Durchmessers für die Chromosphäre. Wenn das Verhältniss des Durchmessers der letzte. ren zum Durchmesser des Kerns wächst, zieht sich aber der von Herrn Halm betrachtete nicht aufgehellte Theil des Absorptionsbandes zu einer schmalen, nach Violett um mehr als die halbe Breite des hellen Bandes verschobenen Linie zusammen, und seine Breite erreicht, wenn der Durchmesser der Chromosphäre z. B. das Vierfache des Durchmessers des Kerns beträgt, nur noch r.6 Procent der Breite des hellen Bandes. Da sich derartige Linienpaare selbstverständlich nicht mit den Doppellinien im Spectrum des Sterns vergleichen lassen, so schien mir auch ohne die mir von Herrn Halm empfohlenen ausführlicheren Darlegungen die geringe Wahrscheinlichkeit der Hypothese hinreichend dargethan zu sein. Besser würde letztere das zweite Entwickelungsstadium des Spectrums darstellen, wenn nur die kräftigste Absorptionslinie in.Betracht gezogen werden könnte. Allein die Auflösung der Absorptionsbänder in Liniengruppen macht hier die Hypothese von vornherein nicht anwendbar. Das Auftreten dieser Liniengruppen dürfte vielmehr der Beginn der Wandlungen sein, welche das Novaspectrum bis zur Aus. bildung der Liniengruppen durchläuft, welche sich an der Stelle der typischen Nebellinien befinden. Zum Verständniss dieser Vorgänge wäre vor allem eine genaue Kenntniss des Spectrums der Nebelmaterie und seiner Beziehungen zum Wasserstoffspectrum erforderlich. 